\title{
Alexander's disease: case report including histopathological and electron microscopic features
}

\author{
G. COLE, F. DE V ILLIER S, N.S.F. PROCT OR, \\ I. F R E I M A N, A N D P. B I L L \\ From the Department of Anatomical Pathology, Neuropathology Division, School of Pathology, \\ South African Institute for Medical Research and University of the Witwatersrand, and the \\ Departments of Neurology and Paediatrics, Baragwanath Hospital, Transvaal, South Africa
}

SUMMARY We report a case of Alexander's disease in a black South African boy of 9 years. The child presented with a grossly abnormal stooped posture, generalised weakness, and slurred nasal speech. Computerised tomography revealed diffuse low radio-density confined to the white matter, and enlarged ventricles. Cerebral biopsy confirmed the diagnosis. The histopathological and electron microscopic features are essentially the same as those described in previous reports. We note the presence of dense clumps of material within some of the Rosenthal fibres, and can find no reference in the literature describing this. Sural nerve biopsy was normal.

\section{Case report}

A black South African boy child aged 9 years and 5 months was first seen at Baragwanath Hospital in July 1978. A rather inadequate history was supplied by his sister. His main complaints were difficulty in walking because of painful knees and backache, and generalised weakness for approximately one month. The patient has six healthy siblings, and his own birth and milestones were apparently normal. From the clinical appearance of the child we considered that he had been ill for longer than the history indicated.

$\mathrm{He}$ was a thin black male child with a head circumference of $570 \mathrm{~mm}$. He had a strikingly abnormal stooped posture, with flexion of the neck, drooping of the shoulders, and flexion of both hip and knee joints (Fig. 1). His speech was indistinct, nasal, and slurred. Pupils were equal in size with a normal response to light and accommodation, and fundoscopy was normal Lack of voluntary movement of the palate and pharyngeal fauces was noted, together with a poor gag reflex. Tongue movements were normal, and no fasciculation was present. Power was decreased in all muscle groups. Tone was within normal limits. Deep tendon and superficial reflexes were normal, and plantar responses were flexor. Sensation was intact for all modalities. There was no

Address for reprint requests: Dr G. Cole, Department of Anatomical Pathology, PO Box 1038, Hospital Street, Johannesburg 2000, South Africa.

Accepted 16 January 1979

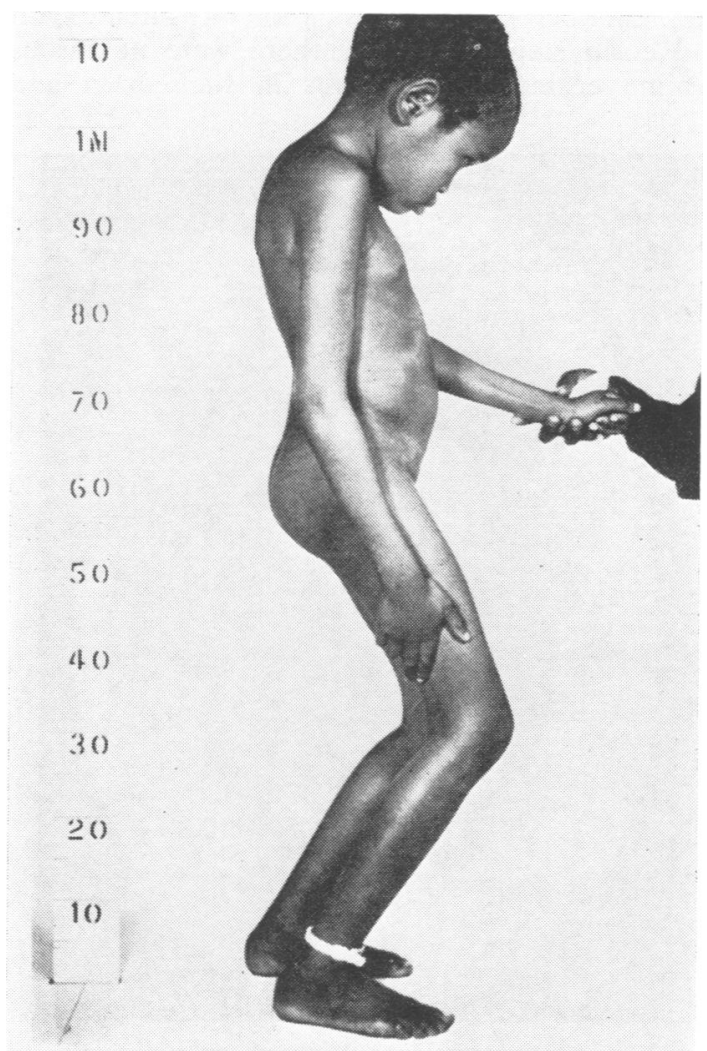

Fig. 1 The patient, with stooped posture, and flexion of neck, hips, and knees. 
evidence of incoordination, nystagmus, or ataxia. This child's mental status was difficult to assess, but he appeared rather dull and listless. The systematic examination was normal. Examination of the cerebrospinal fluid, full blood count, blood urea, electrolytes, plasma proteins, liver function, and creatinine phosphokinase gave normal results. The serum lactic dehydrogenase and alkaline phosphatase levels were minimally raised. Urinalysis was normal. A CAT scan revealed marked dilatation of the lateral and third ventricles and diffuse symmetrical low density, confined to the white matter but more marked in the frontal regions (Fig. 2). These features were strongly suggestive of a demyelinating disease.

\section{HISTOPATHOLOGY}

The brain biopsy specimen consisted of cortex and white matter from the frontal lobe and measured $5 \mathrm{~mm}$. Half the specimen was fixed in phosphate buffered glutaraldehyde for electron microscopic study, and the remainder was fixed in $10 \%$ formol saline. The sections were embedded in paraffin wax. On microscopic examination of haematoxylin and eosin stained sections there were numerous hyaline eosinophilic deposits in the cortex and

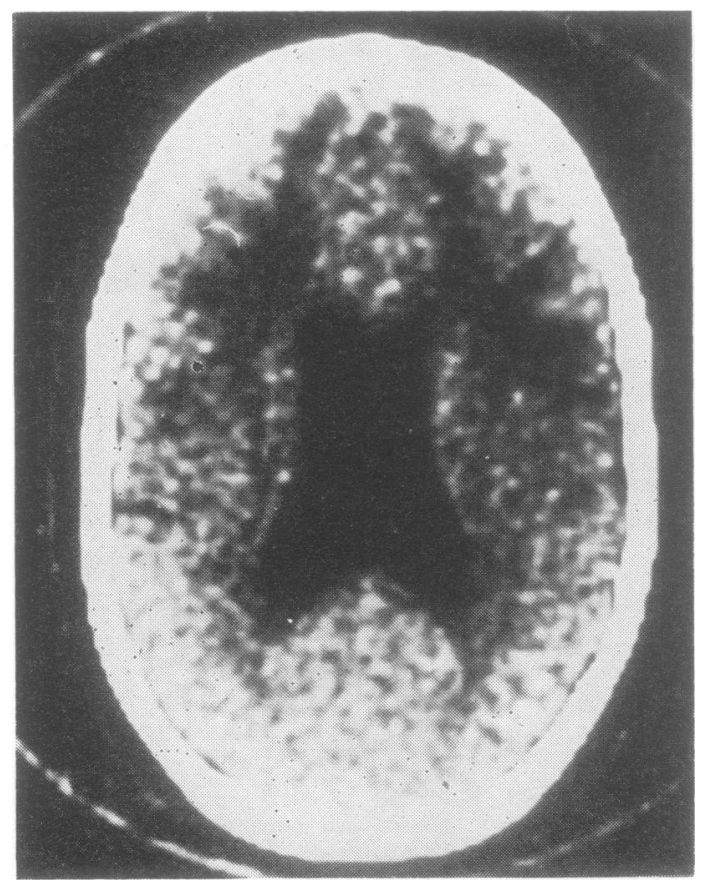

Fig. $2 C A T$ scan showing enlarged ventricles, and low density in the region of the white matter, especially in the frontal area. white matter, particularly in the white matter, and an increased concentration was present in a subpial and perivascular situation. These bodies were homogeneous, varied in shape and size, and were identified as Rosenthal fibres. They lay free in the parenchyma, and were not associated with phagocytic activity. They stained deep purple with phosphotungstic acid haemotoxylin (Fig. 3), and with Masson's trichrome stain they stained deep red. They did not stain with the periodic acid Schiff stain, and stains for iron, calcium, fat, and amyloid. The Kluver-Barrera stain for myelin demonstrated a marked decrease in stainable myelin with a loss of myelinated fibres, while the Rosenthal fibres stained positively with a blue colouration. A brilliant yellow fluorescence was evident with thioflavin T. Gliosis was present in the affected white matter, and swollen, reactive astrocytes were evident. Silver stains for axons showed some axonal loss, and occasional globular thickenings. The Rosenthal fibres were argyrophilic, and the bromsulphalein test for protein (Silverman and Glick, 1965) produced a positive deep blue colouration within them. The staining and histochemical properties in this case of Alexander's disease do not differ essentially from other reported cases (Vogel and Hallervorden, 1962; Friede, 1964; Herndon et al., 1970; Russof et al., 1976). A biopsy sample was taken from the sural nerve. Part of the specimen was fixed in phosphate buffered glutaraldehyde for electron? microscopic examination and the remainder was fixed in $10 \%$ formol saline and in Fleming's solution. The tissue was embedded in paraffin, cut at $4 \mu \mathrm{m}$, and stained with haematoxylin and eosin, Holme's Luxol fast blue, and Masson's trichrome. Microscopic examination revealed well myelinated nerve fibres. The axons appeared normal.

\section{ELECTRON MICROSCOPY}

The brain tissue and sural nerve were fixed in phosphate buffered glutaraldehyde, post-fixed in osmium tetroxide, and embedded in Spierr's resin. The most prominent feature in the brain tissue on electron microscopic examination was the presence of numerous dense, slightly granular, osmiophilic deposits which varied considerably in size, possessed no limiting membrane and corresponded to the Rosenthal fibres seen on light microscopy. In every instance these deposits were surrounded by glial fibres (Fig. 4). This has been observed consistently in other reports, and serves to confirm that these deposits only occur within the cell body or processes of the astrocyte. They have not been reported in any other situation. The glial fibres are invariably fragmented or broken up at the 


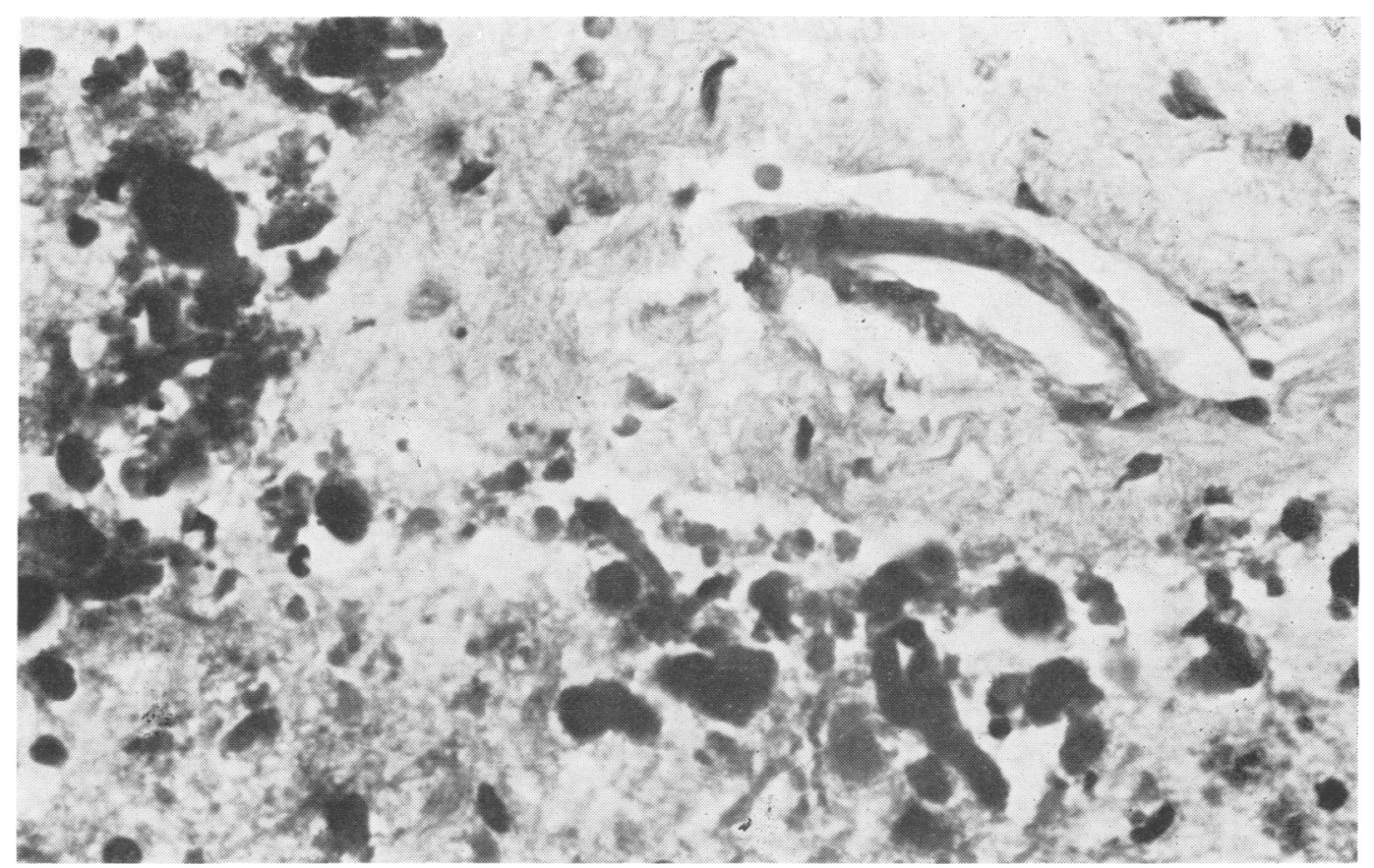

Fig. 3 White matter. PTAH $\times 400$. Rosenthal fibres stain dense purple with PTAH. Note perivascular situation.

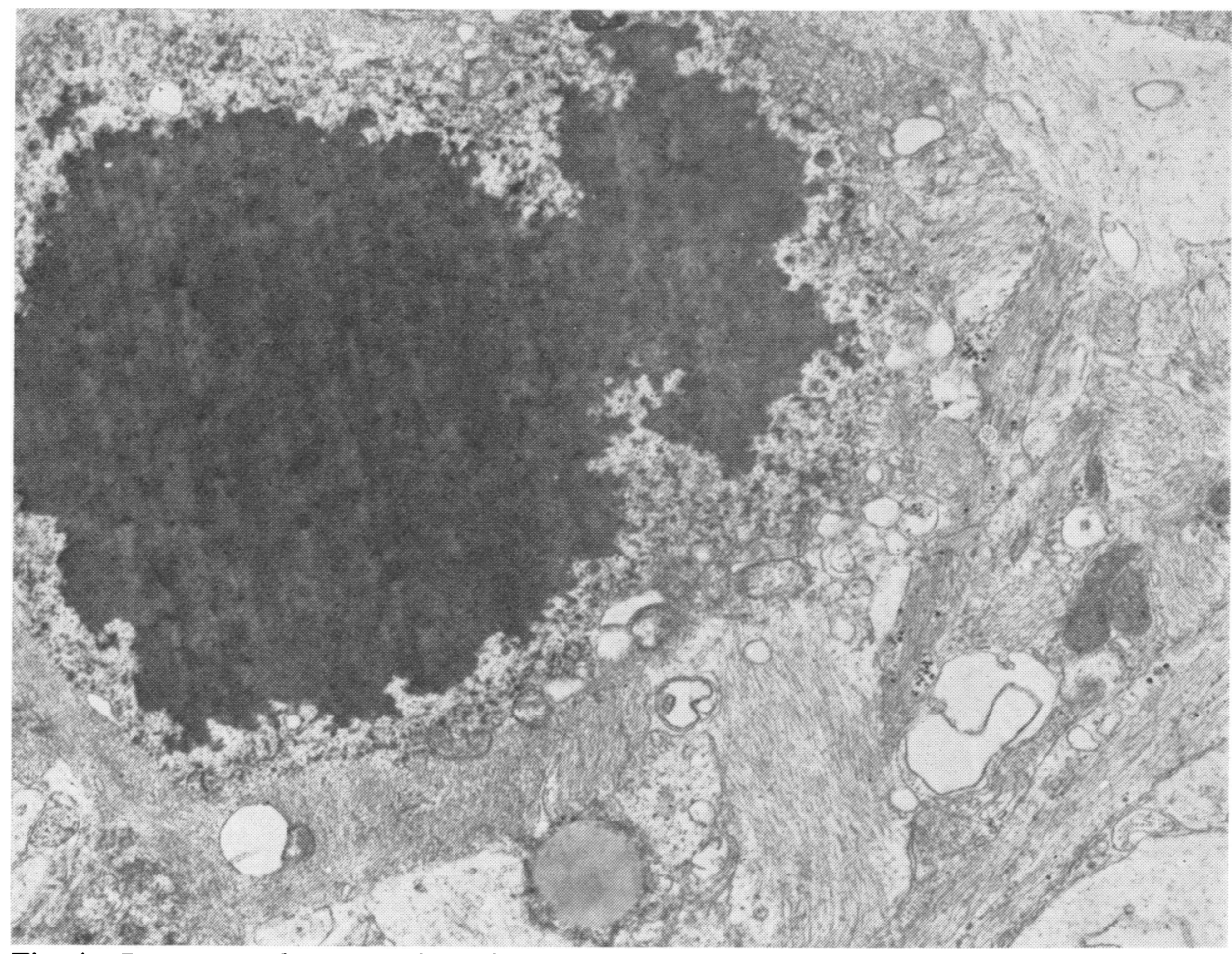

Fig. 4 Dense granular osmiophilic deposit is visible. There is no limiting membrane and it is surrounded by glial fibres which are incorporated in the mass.Vacuoles are seen in relation to mitochondria and within glial fibres. $\times 15700$. 
periphery of the osmiophilic mass. The glial fibres were often thickened, and in some instances this was very marked and they were seen as dense fascicles of fibres (Fig. 5, bottom left). Mitochondria were present in the vicinity of the Rosenthal fibres, and some of them were unusually electron-dense. It has been pointed out by Herndon et al. (1970) that some of the mitochondria appear to be actually incorporated within the electron dense mass, and this is apparent in Fig. 4. Occasional enlarged and abnormal mitochondria were present. Close to a Rosenthal fibre a very abnormal, and extremely electron-dense mitochondrion is present within an axon terminal, which also includes three other mitochondria and synaptic vesicles (Fig. 5, bottom centre). A number of clear vacuoles are seen in relationship to the mitochondria and within the glial fibres (Fig. 4). Extremely dense, very dark irregular clumps of material can be seen within some of the Rosenthal fibres (Fig. 5). We cannot find a reference to similar material in other electron microscopic studies of this disease, and it is uncertain whether this is merely thickened clumps of the same composition as the rest of the Rosenthal fibre or a different substance. Few myelinated nerve fibres were seen in the white matter, but where myelin was present it appeared to be normal.

The appearance of the sural nerve on electron microscopy did not reveal any pathological features (Fig. 6), and normal myelin sheaths, axons, Schwann cells, mitochondria, and collagen were identified.

\section{Discussion}

A description of this disease was first published by Alexander (1949). Over the past 29 years the relatively few reports of similar cases indicate the rarity of this condition. Clinical reports of 11 cases have resembled the original case described by Alexander, occurring in early infancy, and commonly presenting with enlargement of the head, frequently a history of seizures, defective mental development, and spastic paresis. The reported staining reactions and histopathological findings in the brain have shown little variation,

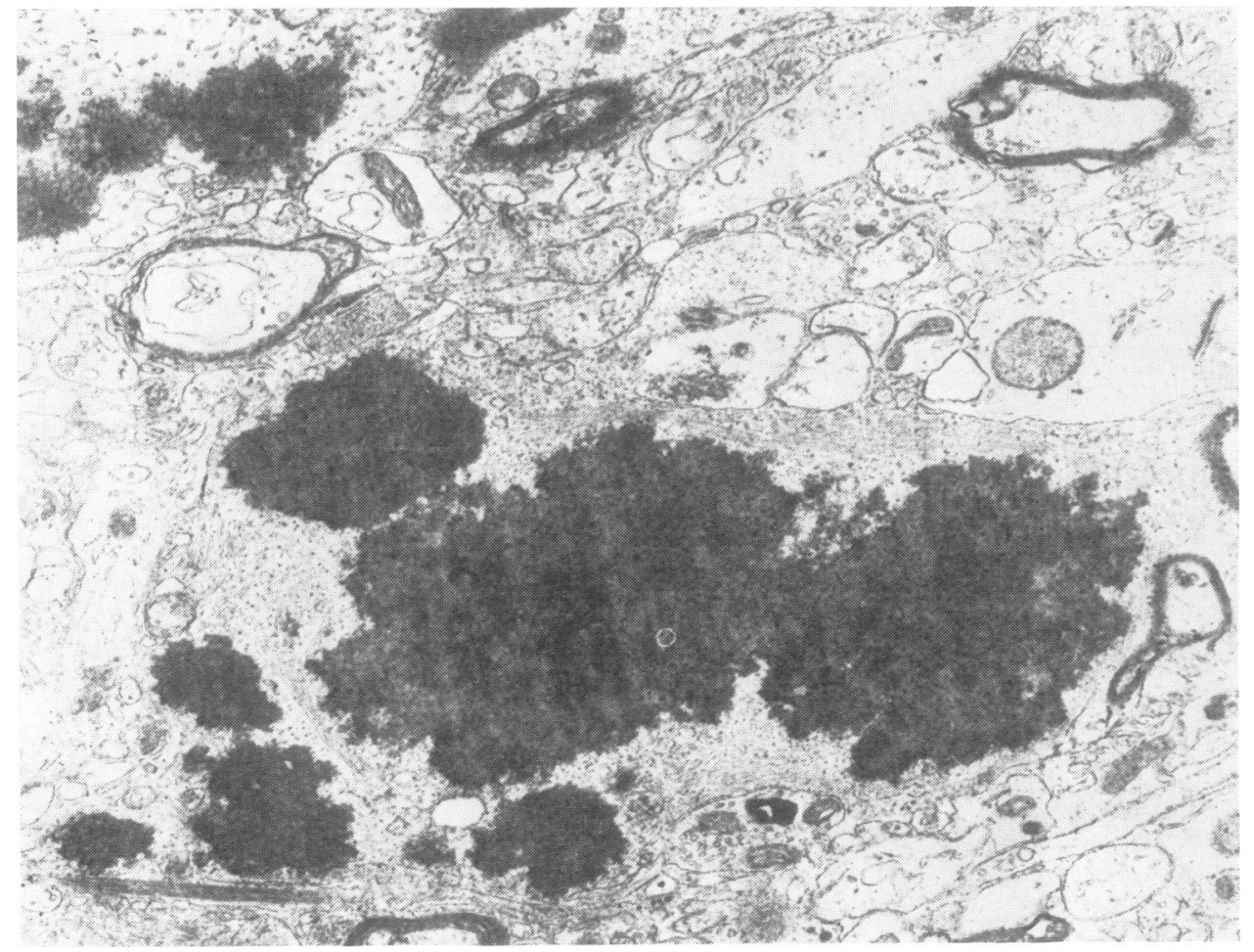

Fig. 5 Axon terminal (bottom centre) contains an abnormal, very dense mitochondrion. A dense collection of glial fibres is present at bottom left. Dark irregular clumps are present within the Rosenthal fibre. $\times 15700$. 


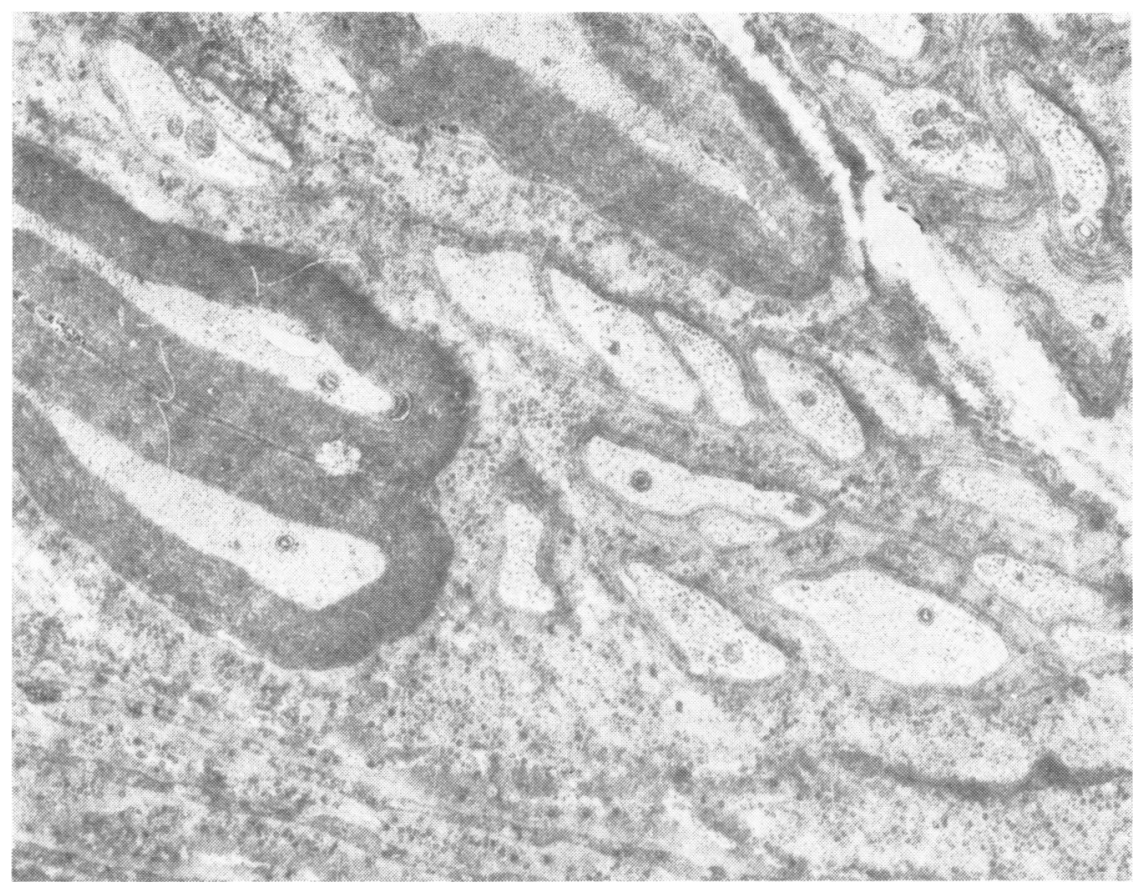

Fig. 6 Section of normal sural nerve $\times 14000$.

the main findings being a dense accumulation of eosinophilic, amorphous deposits both in the cortex and white matter (but denser in the latter) with accentuation in the subpial, subependymal, and perivascular sites. Demyelination is present, and usually quite extensive. Gliosis is seen in the affected areas, and phagocytic activity is notably absent. The eosinophilic bodies are identical to Rosenthal fibres, and the histochemical reactions of the Rosenthal fibres have been similar in all cases with the exception of PAS positivity reported by Wohlwill et al. (1957). Apart from those cases presenting in early infancy, a juvenile group develops symptoms between the ages of 7 and 14 years with progressive bulbar weakness, varying spasticity, and ataxia. Mental abnormality is stated by Russo et al. (1976) to be usually absent, but this would seem likely to depend on the stage and extent of the disease. Fits are not as common as in the infantile cases. The histopathological and electron microscopic appearances of the brain are essentially the same as in the infantile cases, although demyelination may be less severe. A third group consists of adult cases where Rosenthal fibres tend to be focal and related to gliosis in areas of demyelination. In some of these cases the clinical picture has been identical with multiple sclerosis, and Rosenthal fibres are present only in the gliotic area associated with plaque formation. Other adult cases have shown no neurological abnormality, but at necropsy there has been diffuse Rosenthal fibre formation, and patchy, sometimes minimal myelin loss. Russo et al. (1976) subdivided the adult cases into two groups, one with no abnormality on neurological examination, and another presenting as multiple sclerosis. They classified the reported cases up to 1976, and placed 11 cases in the infantile group, five in the juvenile group, and seven in their adult subgroups (four with features identical to multiple sclerosis, and three with no neurological signs). Rosenthal fibres are generally accepted to be derived from degenerating reactive glial fibres, and electron microscopic studies have shown that the Rosenthal fibres are confined to the cell body or processes of astrocytes. In agreement with most observers, Russell and Rubinstein (1977) identify them as irregular osmiophilic bodies, separate from but in places merging into thickened glial filaments. Rosenthal fibres have been found in a variety of disease processes in association with longstanding reactive gliosis, and are commonly found in pilocytic astrocytomas (Russell and Rubinstein, 1977). Alexander's disease is usually attributed to a metabolic defect within the astrocyte, and it is tempting to postulate an enzyme 
deficiency. As suggested by Herndon et al. (1970), the diffuse production of Rosenthal fibres in Alexander's disease may be related to an overproduction of glial filaments with overloading of the degradative pathway, or a defect within the degradative pathway itself. They suggest that a primary metabolic abnormality in the astrocyte becomes manifest at the time of myelination in the infantile cases, and that in addition the defective astrocyte cannot adequately fulfil its normal supportive and metabolic role in relation to the neurone and the oligodendrocyte. Consequently, there is defective myelin formation as a secondary phenomenon. In the cases of later onset, they suggest that the metabolic defect is incomplete, with a delay in the onset after myelination, and therefore myelin loss is not so severe. In the adult cases the astrocytes are unable to handle the increased metabolic turnover which is required in the presence of an increased gliosis, however caused. A similar latent metabolic abnormality is suggested in those cases otherwise identical to disseminated sclerosis. We agree with this attractive hypothesis but, in view of the fact that a localised Rosenthal fibre formation may occur at the site of a longstanding reactive gliosis and is commonly seen within a pilocytic astrocytoma, we question whether some of the adult cases, which differ considerably from the infantile and juvenile cases, should really be included as Alexander's disease. In the same way that, for example, the development of a neurofibrillary tangle is associated with diverse diseases and conditions, so may the Rosenthal fibre develop in a localised area of the brain in circumstances other than Alexander's disease. The absence of sensory symptoms in all cases, the involvement of cranial nerve nuclei concerned with motor function, and the involvement of motor pathways prompted Russo et al. (1976) to postulate that Alexander's disease may be specific to the motor system. They also suggest that if, indeed, there is a deficient enzyme responsible for the disease, it could be that the differences in the infant, juvenile, and adult states are reflected by heterc- and homozygous states. The findings in this first case reported of a black South African child do not differ in any way from previously reported cases. The peripheral (sural) nerve biopsy specimen was normal, and helps to confirm that this disease is confined to the central nervous system. The astrocyte is present only in the brain and spinal cord, while in the peripheral nerve the Schwann cell presides. This perhaps adds further credence to the hypothesis attributing the basic cause of this disease to malfunction of the astrocyte. The patient is still in hospital at the time of writing this report, and his condition is deteriorating. He is more listless, and can do less for himself than on admission. The stooped posture is much worse, and he walks with his knees bent to a very marked degree. This is one of the few cases where the diagnosis of Alexander's disease has been made during life, and clinical observation of this unfortunate child continues.

We would like to thank $\mathrm{Mr} R$. R. Hill of the Department of Histopathology and Electron Microscopy for the preparation of photomicrographs.

\section{References}

Alexander, W. S. (1949). Progressive fibrinoid degeneration of fibrillary astrocytes. Brain, 72, 373-381.

Friede, R. (1964). Alexander's disease. Archives of $\overrightarrow{-}$ Neurology (Chicago), 11, 414-422.

Herndon, R., Rubinstein, L., Freeman, J., and Mathieson, G. (1970). Light and electron microscopic observations on Rosenthal fibres inco Alexander's disease and in multiple sclerosis. Jour-? nal of Neuropathology and Experimental Neurology, 29, 524-551.

Russell, D., and Rubinstein, L. (1977). Pathology of Tumours of the Nervou; System, 4th edition, p. 161. Edward Arnold: London.

Russo, Jr., L. S., Aron, A., and Anderson, J. (1976). Alexander's disease: a report and reappraisal. Neurology (Minneapolis), 26, 607-614.

Silverman, L., and Glick, D. (1965). Studies in histochemistry. Histochemical demonstration of protein with bromsulfalein. Journal of Histochemistry and Cytochemistry, 14, 425-426.

Wohwill, F., Bernstein, P., and Yakovlev, P. (1957). Case of unusual presumably familial leukodystrophy with megalencephaly. Journal of Neuropathology and Experimental Neurology, 16, 129-130.

Vogel, F. S., and Hallervorden, J. (1962). Leukodystrophy with diffuse Rosenthal fibre formation. Acta Neuropathologica, 2, 126-143. 